\title{
An Integrated System for Multichannel Neuronal Recording with Spike / LFP Separation, Integrated A/D Conversion and Threshold Detection
}

\author{
Yevgeny Perelman and Ran Ginosar \\ VLSI Systems Research Center \\ Department of Electrical Engineering \\ Technion-Israel Institute of Technology, Haifa 32000, Israel \\ perelman@tx.technion.ac.il, ran@ee.technion.ac.il \\ Tel: 972-4-8294697 Fax: 972-4-8295757
}

\begin{abstract}
A mixed-signal front-end processor for multichannel neuronal recording is described. It receives twelve differential-input channels of implanted recording electrodes. A programmable cutoff HPF blocks DC and low frequency input drift at about 1Hz. The signals are band-split at about $200 \mathrm{~Hz}$ to low-frequency local field potential (LFP) and high-frequency spike data (SPK), which is band limited by a programmablecutoff $L P F$, in a range of $8-13 \mathrm{kHz}$. Amplifier offsets are compensated by 5-bit calibration DACs. The SPK and LFP channels provide variable amplification rates of up to 5000 and 500 , respectively. The analog signals are converted into 10 -bit digital form, and streamed out over a serial digital bus at up to 8Mbps. A threshold filter suppresses inactive portions of the signal and emits only spike segments of programmable length. A prototype has been fabricated on a $0.35 \mu \mathrm{m}$ CMOS process and tested successfully, demonstrating a $3 \mu \mathrm{V}$ noise level. Special interface system incorporating an embedded CPU core in a programmable logic device accompanied by real-time software has been developed to allow connectivity to a computer host.
\end{abstract}

\section{INTRODUCTION}

Availability of multi-site neuronal electrodes, such as the Michigan probe [1] or the Utah array [2], has enabled the development of highly integrated multi-channel recording devices with large channel counts. These devices are of importance to various aspects of neurophysiological research [3], [4], [5].

Multi-site electrodes can potentially provide for simultaneous monitoring of hundreds and even thousands of neurons. The raw data rates that are generated by such populations are large [6]. When sampled at 20Ksps with eight bit precision, a hundred electrodes would generate raw data rate of 16Mbps. Communicating such volumes of neuronal data over batterypowered wireless links while maintaining reasonable battery life is hardly possible with common methods of low-power wireless communications. Evidently, some form of data reduction must be applied. One possible way is to utilize some form of lossy data compression to reduce the raw waveform data capacity. A method employing Wavelet Transform was suggested in [7]. Alternatively, one might extract the significant

\footnotetext{
${ }^{0}$ Copyright (c) 2006 IEEE. Personal use of this material is permitted. However, permission to use this material for any other purposes must be obtained from the IEEE by sending an email to pubs-permissions@ieee.org.
}

features of the neuronal signal and limit the transmitted data to those features only. For example, it is possible to detect the presence of neuronal spikes as demonstrated in [6] and communicate only active portions of recorded signals, which may lead to an order of magnitude reduction in the required data rate [8]. Another order of magnitude reduction can be achieved if the neuronal spikes are sorted on the chip and mere notifications of spike events are transmitted to the host. Power feasibility of on-chip spike sorting with common sorting algorithms that are usually software-based is verified in [9]. Adapting these algorithms for utilization in VLSI can yet lead to significant power savings, with only minor sacrifice of results accuracy [10], [11]. In [12], it is suggested to measure and communicate certain features of the incoming spikes; the spike sorting can subsequently operate on these features.

Our research is aimed at an integrated wireless recording device capable of acquiring neuronal activity over a large number of channels, digitizing, performing data reduction and communicating over a bi-directional wireless link. This paper describes a multi-channel neuronal recording frontend integrated circuit, fabricated in $0.35 \mu \mathrm{m}$ standard CMOS process. The front-end acquires neuronal signals from twelve true-differential recording channels, performs analog signal conditioning including separation of spike and Local-FieldPotential frequency bands, digitizes the outputs and transmits the data to the host over a serial bus. An on-chip controller provides a level of data reduction by thresholding the incoming signals and transmitting only the "active" signal portions, i.e. segments of signals immediately following threshold crossing events. The front-end is to be integrated with spike-sorting hardware and wireless modem on the PCB level prior to full VLSI integration.

Separating the LFP and the SPK bands at the analog portion of the frontend may have certain advantages, as it reduces the dynamic range requirements on the last frontend stages: In a signal recorded by an extracellular microelectrode, neuronal firing activity occupies the $100-10,000 \mathrm{~Hz}$ frequency band; its amplitude is typically lower than $500 \mu \mathrm{V}$. The Local Field Potential (LFP) occupies the lower frequencies, below $100 \mathrm{~Hz}$, with amplitudes below $5 \mathrm{mV}$. The signal-to-noise ratio of the combined signal is rather large; as the microelectrode 
noise [13] and background noise of cortical activity [14] are typically $5 \mu \mathrm{V}$, it may reach $60 \mathrm{~dB}$.

Since the LFP must be filtered out prior to spike sorting, it is possible to block it right at the front-end [15], by highpass filtering below $100 \mathrm{~Hz}$. It is commonly indicated, however, that LFP carries important information [16], [17], [18]. Thus the recording device should preferably make this information available together with the spike data. Several front-end circuits pass the LFP band intact: [19], [20], [21]. They block the large input DC offsets, typical of neuronal signals, by high-pass filtering below $1 \mathrm{~Hz}$. As the entire combined signal is passed, the minimal required precision of subsequent data acquisition is 10 bit, defined by the signal SNR. The maximal gain is limited by the LFP magnitude and chip supply voltage. Since the firing activity (SPK) has ten times lower magnitude than the LFP, it can be amplified only to one tenth of the output swing.

Splitting the signal into two bands after the first amplification stage allows separate processing of the LFP and SPK bands, amplifying both to the full swing. Consequently, the system dynamic range needs only be a 100 , as determined by the SNR of the SPK signal, and no more than seven bits data acquisition is required.

The chip architecture is described in Sec. II. A special embedded system design for interfacing the chip is briefly described in Sec. III. Recording channel circuitry is reviewed in Sec. IV, with selected test results brought in Sec. V. Section VI summaries the discussion.

\section{ARchitecture}

The chip architecture is shown in Fig. 1. The on-chip controller is responsible for host communication, chip timing, internal register access, channel readout and spike detection. Channel registers and A/D converters are accessed through an internal parallel bus, mastered by the controller.

The controller has two modes of operation, programming and streaming. In the programming mode, contents of internal registers can be stored and fetched by the host. In the streaming mode, the controller continuously polls the channel ADCs, checks for threshold crossing events on every channel, and transmits the active signal segments to the host. All twelve channels or an arbitrary subset thereof can be enabled for data streaming.

A threshold crossing event is triggered for a certain channel when the output of this channel falls below the low threshold or rises above the high threshold. The controller polls continuously the ADC outputs to check for threshold crossing events. A certain number of samples from that channel is communicated to the host following the threshold crossing event. The threshold values and the number of samples to transmit after the threshold event are programmable. The entire data stream, without clipping, can be obtained from the chip by setting both thresholds identical.

\section{Chip Communications}

The chip communicates over a McBSP [22] bus. This is a five-wire, full-duplex, bit-serial synchronous bus; a synchronization clock signal is constantly supplied by the host. The communication is carried out in frames; the host sends 24-bit frames (we refer to this direction as downwards) and the chip replies with 16-bit frames (the upwards direction). The lengths of downward and upward frames were conveniently chosen to match the lengths of a single host instruction packet and a single reply packet respectively.

The maximal data rate that is generated by the chip can be calculated as follows: A channel A/D sample is ten bit wide (although seven bits are sufficient, we have implemented ten bit ADCs for verification purposes). Together with a four bit channel number and a two bit control field, an A/D sample can be communicated in a single 16-bit frame. With SPK channel sampled at $40 \mathrm{Ksps}$, a single channel would generate a $640 \mathrm{Kbps}$. The LFP channel needs to be sampled with a much lower rate (1Ksps would be enough), with a combined rate of $656 \mathrm{Kbps}$. Although there are only twelve channels in the current version of the chip, the bus interface was designed to support sixteen channels for future versions; the aggregate datarate is therefore $10.5 \mathrm{Mbps}$. The bus was set to operate on a slightly higher, $12.5 \mathrm{MHz}$ clock signal.

\section{Instruction Set and Register Access}

The chip operation is controlled through instructions sent via the McBSP bus. Four instructions are available:

- STORE reg val: Store value in a register.

- FETCH reg: Fetch register contents.

- $R U N$ : Start streaming data.

- STOP: Halt streaming data.

There are two kinds of parameters that control the chip, those affecting controller operation and those affecting the channels. The former include clock divider settings, threshold values, number of samples to communicate upon threshold detection and channel enabling bit mask; the registers for their storage reside in the controller and are accessed directly. The latter include offset calibration data, channel gains and filter frequencies; the registers are distributed over the channels and are accessed through the internal bus.

The internal bus has eight data lines, two control lines and a clock. A register connected to the bus is identified by a distinct eight-bit address. Every bus access is carried out in two steps; during the address step (ADDR is high) the address is driven on DTA $<7: 0>$. The register matching this address is selected. During the data step (ADDR is low) the contents of the selected register are driven on the bus by the channel (WR is low) or the register is updated with the value on the bus (WR is high). The bus can be accessed in three possible scenarios, SELECT-READ, SELECT-WRITE and SELECT-READ-MODIFY-WRITE. Fig. 2 shows the bus signals during the last access scenario. A ten-bit A/D sample is read with two register accesses, since the registers are fixed eight-bit width. Another write access is needed to request the next conversion at the channel ADC. Reading a single A/D sample out of a channel takes therefore five clock cycles. 16 channels sampled at $40 \mathrm{Ksps}$ need bus clock rate of at least $3.2 \mathrm{MHz}$. Since the bus clock is derived form the McBSP clock by integer division, the most suitable division factor is 3 , setting the bus clock to about $4.16 \mathrm{MHz}$. 


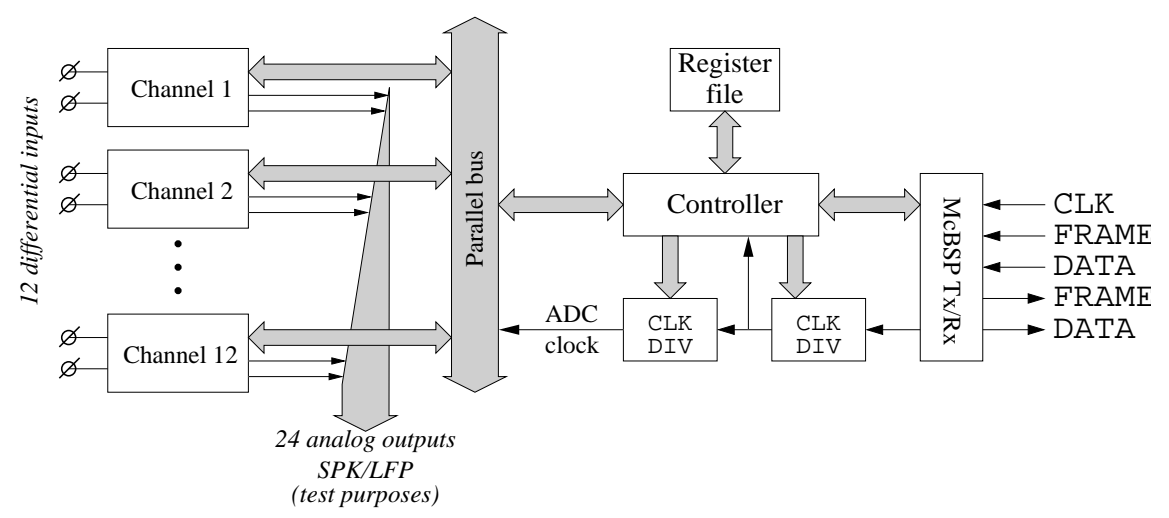

Fig. 1. Chip architecture

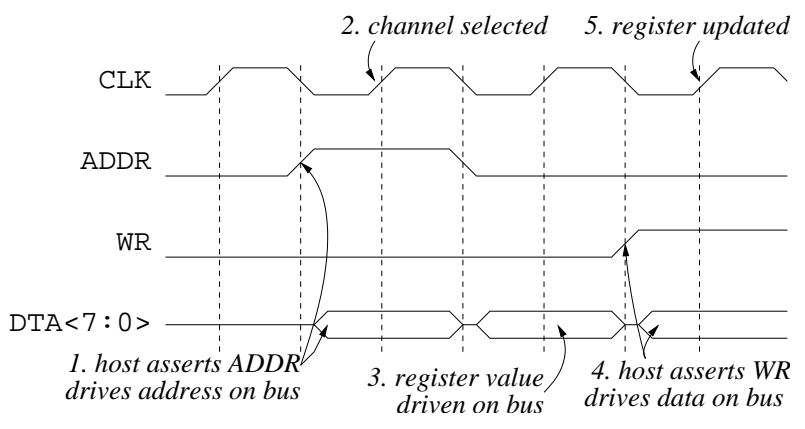

Fig. 2. Internal bus signals during SELECT-READ-MODIFY-WRITE access

\section{Host INTERFACE}

A special interface provides for communication between a personal computer and the neuronal recording front-end. The basis of the interface is an Altera Nios II development kit board incorporating an Altera Cyclone II FPGA device, RAM and flash memory, and an integrated Ethernet physical interface/MAC (Fig. 3).

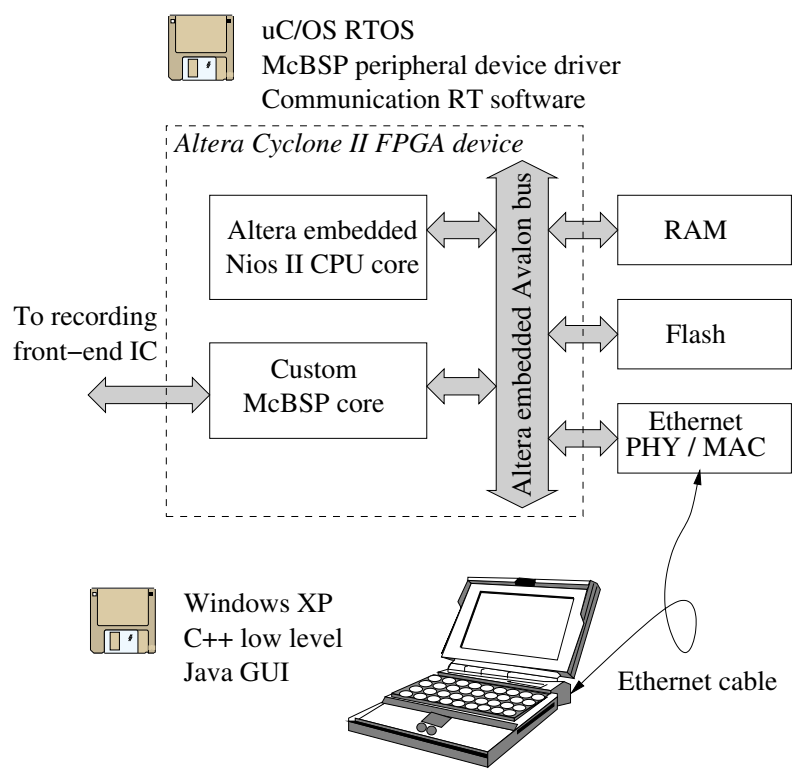

Fig. 3. Host interface

The FPGA incorporates an Altera Nios II embedded processor core (running at 50MHz), bus logic and customdeveloped peripheral for McBSP communications with the neuronal recording front-end. The embedded processor executes the $\mu \mathrm{C} / \mathrm{OS}$ real-time operating system (RTOS) and custom-developed real-time software for handling the neuronal data stream. The software reads the serial McBSP data, packetizes it and transmits the packets over Ethernet to a host computer using UDP/IP protocol. It also handles the incoming instructions from the host and communicates them to the chip.

The host side software consists of a low-level C++ module that handles the data stream in real time, dumps it onto the disk and performs the decimation necessary for an onscreen display. Displaying data on screen without some sort of decimation (i.e. downsampling) would result in too high screen refresh rates, imperceivable by the human eye. Data display and system control are performed by the top-level Java GUI module (Fig. 4).

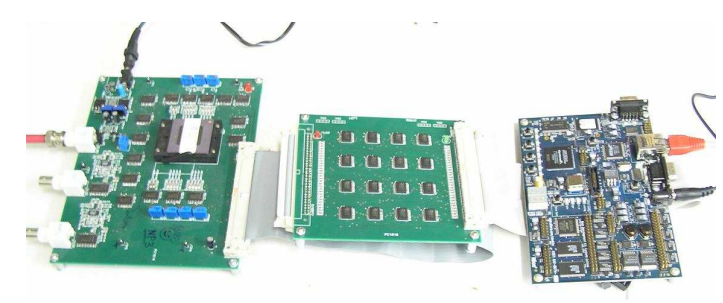

(a)

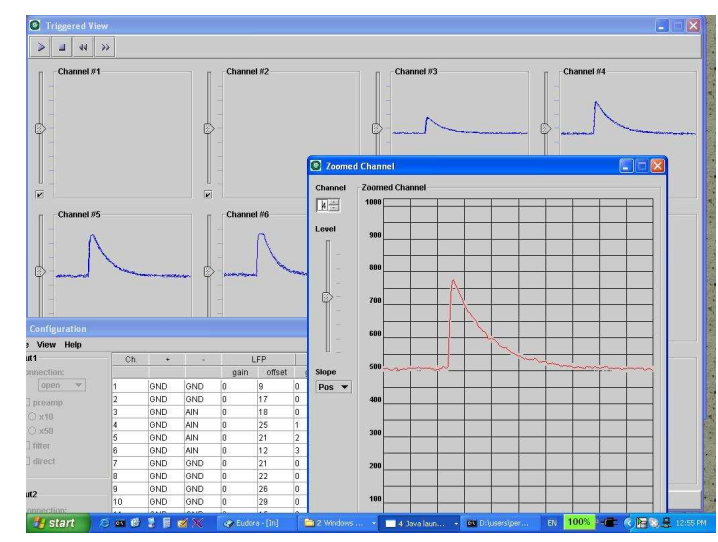

(b)

Fig. 4. (a) Test setup. Boards from left to right: evaluation, digital signal buffering and the embedded system. (b) Screenshot 


\section{RECORDING CHANNEL}

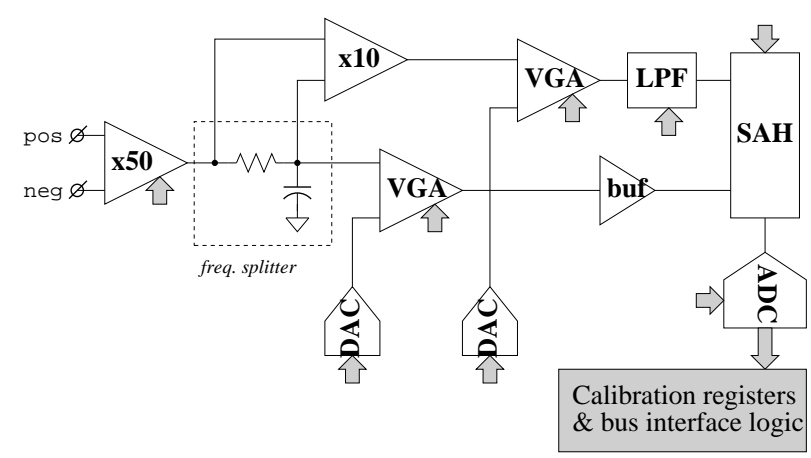

Fig. 5. NPR03 recording channel

Fig. 5 shows the recording channel block diagram, as implemented on a $0.35 \mu \mathrm{m}$ CMOS chip. The input signal is amplified fifty times by the first stage, which also converts the differential signal to single-ended. A first-order RC filter splits the signal into high frequency SPK and low frequency LFP parts. The splitting pole is roughly placed at $200 \mathrm{~Hz}$, with a $5 \mathrm{M} \Omega$ resistor (high resistive polysilicon) and $160 \mathrm{pF}$ (gateoxide) capacitor.

The SPK signal is amplified by an intermediate $\times 10$ stage and a variable gain amplifier (VGA) with digitally selectable gain of $2.5,5,7.5$ or 10 . The SPK chain maximal gain is therefore 5,000. SPK signal band is limited by a second-order Bessel LPF (Fig. 6), implemented as a Sallen-Key biquad [23]. The $-3 \mathrm{~dB}$ frequency is digitally programmable in the range of $8-13 \mathrm{kHz}$, by means of a multi-tap resistor. The LFP signal is amplified by an identical VGA, without the intermediate $\times 10$ amplifier. The LFP chain maximal gain is 500 .

Both SPK and LFP channels have to be compensated for DC offsets introduced by element mismatch. The LFP channel amplifies the preamp input offset (typically hundreds of $\mu \mathrm{V}$ ) by $54 \mathrm{~dB}$; unless compensated, it would severely degrade the LFP dynamic range or even saturate the VGA. The SPK channel amplifies the offset of the intermediate $\times 10$ stage by $40 \mathrm{~dB}$, as the preamp DC is blocked by the splitter. Though smaller than LFP, SPK offset is yet significant: the $\times 10$ stage has larger input offset compared to the preamp, as the latter uses very large input devices (due to noise requirements). DC offset compensation is carried out by adjusting the VGA reference voltages with a pair of 5-bit calibration DACs.

Finally, the channels are multiplexed by a Millercapacitance sample and hold circuit (Fig. 6) and converted by a 10 bit successive approximation ADC, which incorporates a special, low-power inverted-ladder DAC [24].

\section{Input Preamp}

Voltage offsets inherent in neural signal recordings constitute a major challenge in preamplifier design. An input signal must be high-pass filtered at frequencies as low as several Hertz, to let the LFP signal pass unsuppressed. Such time constants are not readily available in integrated circuits.

Several approaches for DC offset stabilization have been reported: Off-chip elements are sometimes employed at input

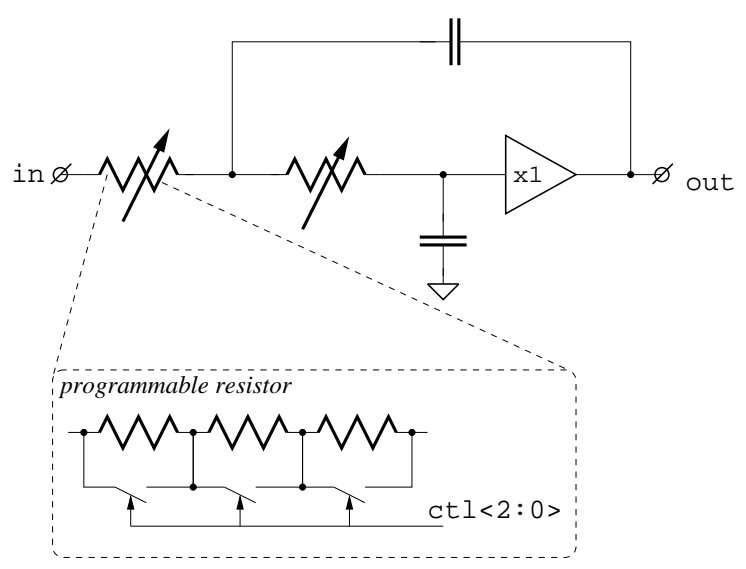

(a)

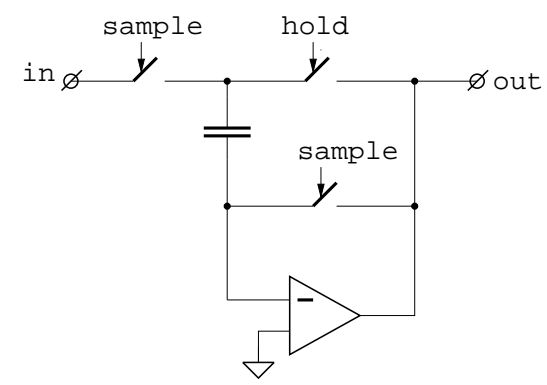

(b)

Fig. 6. (a) 2nd order Sallen-Key biquad (b) Miller capacitance sample-andhold

stages [8], [25]. Several fully integrated approaches were also demonstrated: The signal can be capacitively coupled to the amplifier using the polarization capacitance of the electrode, shunt either by a weak-inversion MOS transistor [21] or a reverse-biased diode [26], both delivering a large small-signal impedance to form a low frequency pole at the input. In the former, the gate bias of the shunting transistor is derived with a laser-trimmed resistor. The DC gain of this scheme is not strictly zero, since the real part of the electrode impedance, although very large, is not infinite. DC gain is therefore defined by the ratio of the shunting resistance and the parallel resistance of the electrode. Another fully integrated approach suggests using a pseudo-resistor device based on a weak inversion MOS and a parasitic bipolar [27], [19], [28], [12]. Such a device has an extremely large small signal resistance at small bias voltages.

The proposed preamp schematic is shown in Fig. 7. A differential stage with a gain of five and an HPF filter is followed by a differential-to-single-ended stage with a gain of ten ${ }^{1}$. The total preamp gain is therefore 50 . The minimal gain to be provided by the preamp is determined by noise constraints as follows. RMS noise introduced by the frequency splitter resistance into the SPK signal (band of $10 \mathrm{kHz}$ ) is:

$$
\sqrt{4 k T R \cdot 10^{4}}=28 \mu \mathrm{V}
$$

Hence, the preamp must provide gain well above 20dB to keep

\footnotetext{
${ }^{1}$ The output impedance of the latter must be low enough to provide a good impedance mismtach with the splitter resistance. The actual output impedance of the $\times 10$ stage is about $100 \mathrm{k} \Omega$.
} 


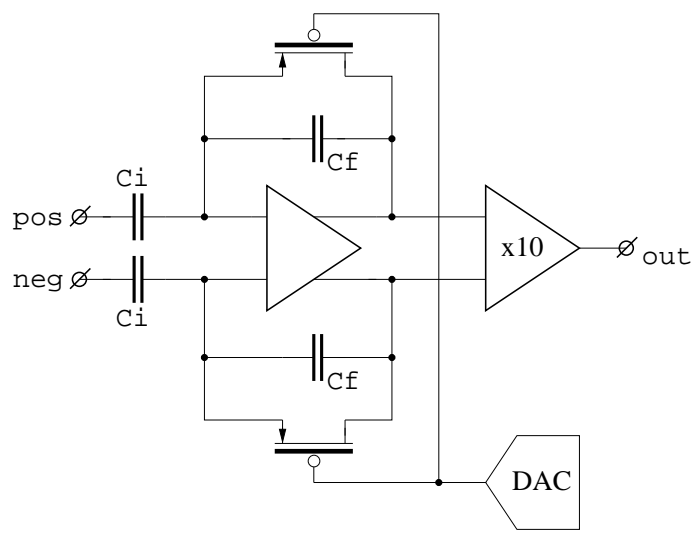

Fig. 7. NPR03 preamp

the splitter contribution below the target $2 \mu \mathrm{V}$.

We have chosen to place a weak inversion MOS transistor in parallel with $C_{f}$, to provide a first order high-pass filter for input DC suppression. The cutoff frequency is digitally programmable through gate bias voltage adjustment with a calibration DAC. As the conductance provided by the feedback transistor does not belong to a set of controlled process parameters, we have measured a significant variability (more than an order of magnitude) in cutoff frequency among the channels, even on the same die (Fig. 8). Being able to control the gate bias voltage, we have managed to calibrate all channels to a $1 \mathrm{~Hz}$ cutoff.

Given a single pole splitter with pole frequency $f_{1}$, the noise energy contributed by the feedback resistor to the SPK signal is:

$$
\int_{0}^{\infty} \frac{4 k T g}{\omega^{2} C_{f}^{2}+g^{2}} \times \frac{\omega^{2}}{\omega^{2}+\omega_{1}^{2}} d \omega
$$

where $g$ stands for the conductance of the DC nulling resistor. Assuming $f_{1}$ (about $200 \mathrm{~Hz}$ ) is much larger than the selected cutoff frequency $f_{0}$ of the input HPF, the expression above can be re-written as:

$$
\frac{k T}{C_{f}} \times \frac{\omega_{0}}{\omega_{1}}
$$

and reflected to the input as:

$$
\frac{k T}{C_{f}} \times \frac{\omega_{0}}{\omega_{1}} \times\left(\frac{C_{f}}{C_{i}}\right)^{2}
$$

Placing the resistive element in the feedback has an important advantage: the noise generated by the resistor is attenuated by the amplifier gain. For $f_{0}$ of $1 \mathrm{~Hz}, f_{1}$ of $200 \mathrm{~Hz}$, first stage gain of five and $C_{f}$ of $500 \mathrm{fF}$, we obtain about $1.8 \mu \mathrm{V}$ input RMS noise (remembering that there are two resistive elements in a differential stage). The calculations do not include the opamp noise.

Another important tradeoff is revealed by the above formula: higher $f_{0}$ yields higher noise contribution of the pseudoresistors and better DC rejection. In that context, providing for a selectable cutoff frequency is another advantage.

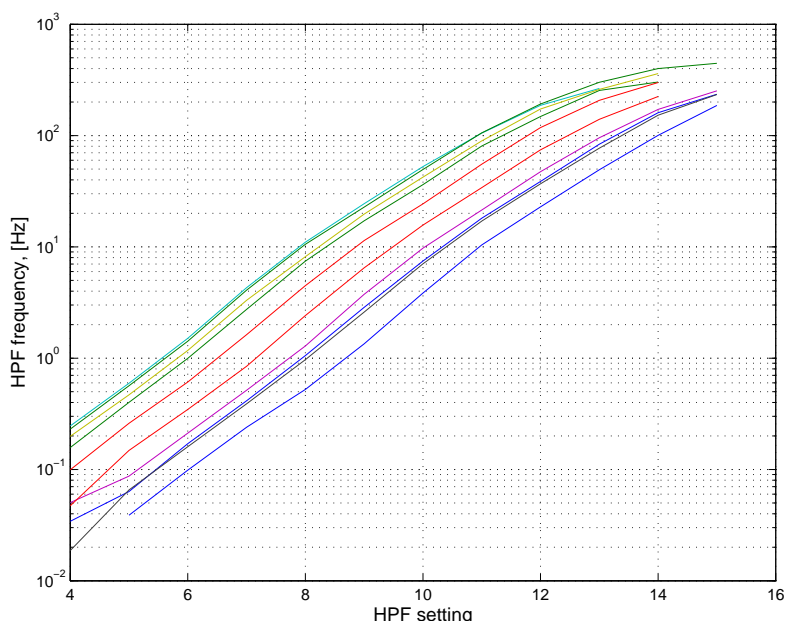

(a)

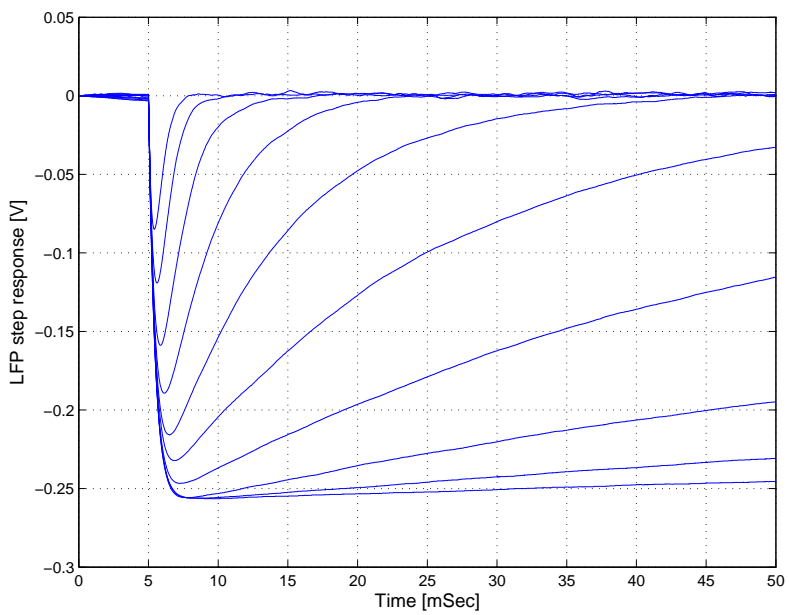

(b)

Fig. 8. (a) Input HPF cutoff vs. DAC setting, several channels; (b) LFP step response for several DAC settings in the time domain

\section{Measurement Results}

A $0.35 \mu \mathrm{m}$ CMOS double poly, quad metal $3.7 \times 3.9 \mathrm{~mm}$ IC (Fig. 9) was fabricated at AustrianMicroSystems and tested electrically.

The electrical tests were carried out on twelve channels from ten different dies. The measurements were completely automated; the instruments and the chip were controlled by MATLAB software.

Small signal responses of the SPK and the LFP channels measured on several dies are presented in Fig. 10. The flatband gains for the SPK and LFP chains were measured as 3780 and 430, respectively. They have small variations over different dies, some 1\% for SPK and 2\% for LFP. The deviation from the target average values is due to an inaccurately predicted gain of the $\times 10$ stages (and VGAs, which have similar configurations), which turned out to be 9.1 instead of 10 . Thus gain errors of $0.91^{3}$ and $0.91^{2}$ are introduced into SPK and LFP chains respectively.

It can be observed (on both SPK and LFP graphs) that the frequency splitter pole varies significantly among different curves. Its average location is also displaced, $350 \mathrm{~Hz}$ instead 


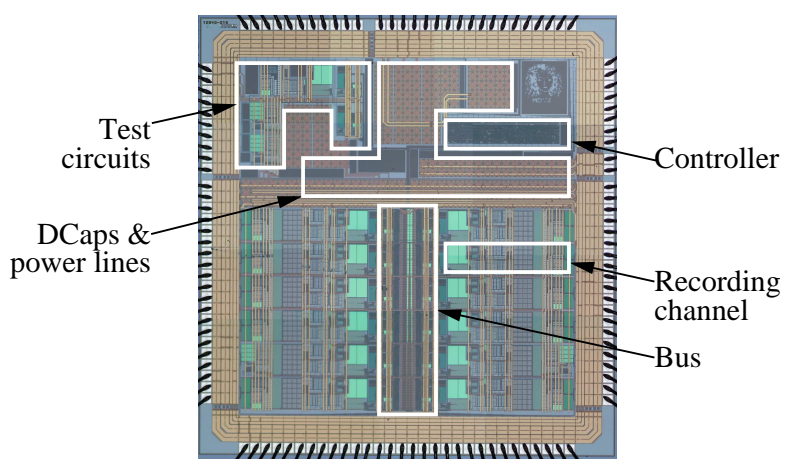

Fig. 9. Die photo

of $200 \mathrm{~Hz}$. This is due to a failure in a bias circuit that was supposed to provide well bias for a large MOS capacitor inside the band splitter.

Fig. 11 shows the SPK channel gain and the cutoff frequency of the output LPF for different settings of digital controls. LPF digital control input determines how many segments are connected in parallel in filter resistors. Thus the control value is directly proportional to the time constant and inversely proportional to the cutoff frequency.

Noise measurements were carried out with grounded inputs. Example results for noise measurements in SPK and LFP channels are presented in Fig. 12, along with simulated curves. The total input-referred noise is $3 \mu \mathrm{V}$ for SPK chain and $10 \mu \mathrm{V}$ for LFP chain, when measured down to frequency of $10 \mathrm{~Hz}$. The low-frequency behavior of the LFP noise is $1 / f^{2}$, and not $1 / f$ as might be expected. This is due to leakage currents through the pseudo-resistor MOS diffusions in the input stage. The area of these diffusions must be kept small in the layout. The input stage of the preamplifier consumes $75 \mu \mathrm{A}$; the corresponding NEF [29],

$$
N E F=V_{\text {noise }, r m s} \cdot \sqrt{\frac{2 I_{d d}}{\pi \cdot U_{T} \cdot 4 k T \cdot B W}}
$$

can be calculated for the bandwidth of $10 \mathrm{kHz}$ as 10.4 . It is possible to design a more efficient amplifier in terms of NEF. An example reported in [19], which also presents a thorough comparison of numerous reported neuronal preamplifiers and their NEF. Although possible, further noise reduction of our preamplifier would require an increase of the input stage area that was not allowed by the overall chip area allocation. The area occupied by the preamplifier is $0.076 \mathrm{~mm}^{2}$, including the $\times 10$ stage and the bias DAC. The overall channel current consumption (including the sample-and-hold and the ADC) is about $1 \mathrm{~mA}$, mostly due to the inefficient $\times 10$ and VGA stages (to be redesigned in future versions). The total chip power consumption is about $12 \mathrm{~mA}$. The 10-bit ADC was designed for a DNL below 1LSB and measured a DNL of 0.8LSB. 1.8LSB INL was also measured. The output voltage range is some $0.5 \mathrm{~V}$ below the supply rails, limited mostly by the SAH circuit. This leads to overall SPK channel dynamic range of 150-600, depending on the selected gain. For a sine output of $1 \mathrm{Vpp}$ amplitude, a THD of below $1 \%$ was measured both on SPK and LFP. PSRR and CMRR of the entire SPK channel have been estimated by simulations as $70 \mathrm{~dB}$ and $90 \mathrm{~dB}$ respectively.

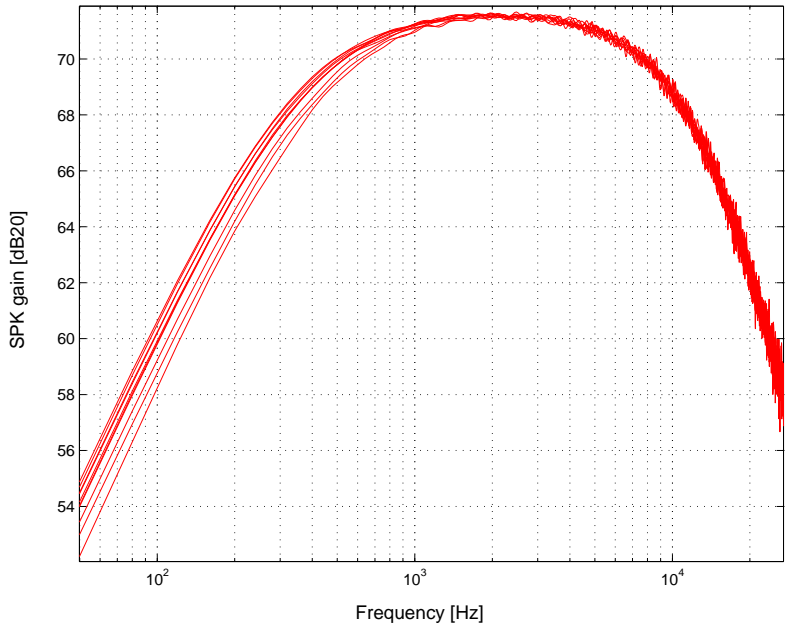

(a)

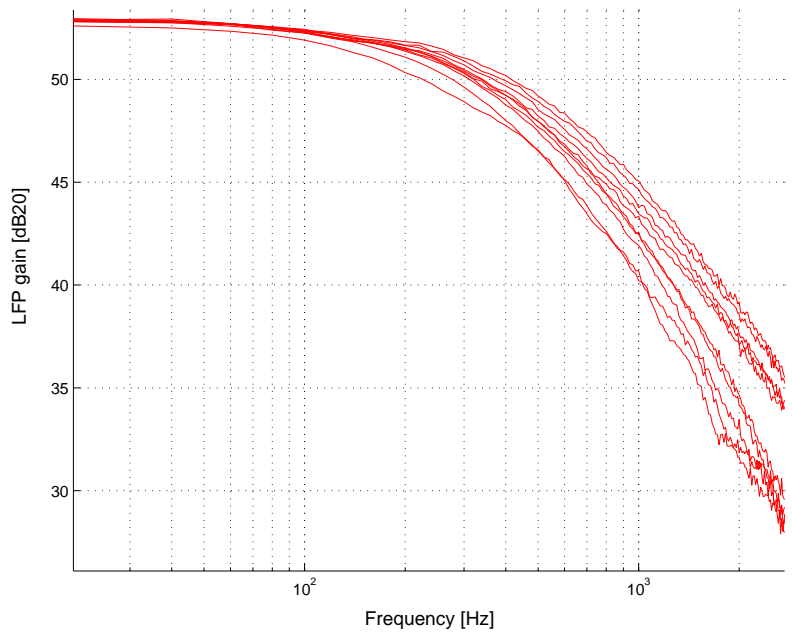

(b)

Fig. 10. Frequency responses: (a) SPK. (b) LFP.

An observation was made during the noise measurements of the chip, regarding a better noise immunity of the fully integrated preamplifier compared to a preamplifier with external capacitors. A preamplifier with external capacitors described in [8] was included on the chip for testing purposes. A noise measurement was performed simultaneously on the integrated preamplifier and the preamplifier with external capacitors (Fig. 13). The circuit with external elements is far more susceptible to external noise sources. We believe that the noise is induced on the discrete capacitors and inherently longer board tracks (due to the presence of capacitors).

The recording system was successfully tested in-vivo with Michigan probes implanted into a cortex of a rat. Samples of recorded signals are shown in Fig. 14.

\section{SUMMARY}

A mixed-signal processor for multi-channel neuronal recording has been presented. It receives twelve differentialinput channels of implanted recording electrodes. The signals are split at about $200 \mathrm{~Hz}$ to low-frequency local field potential 


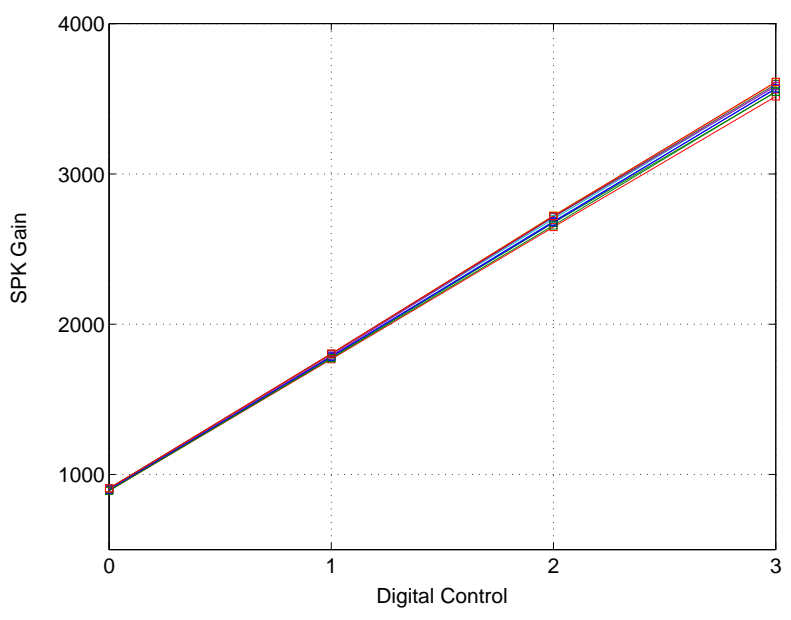

(a)

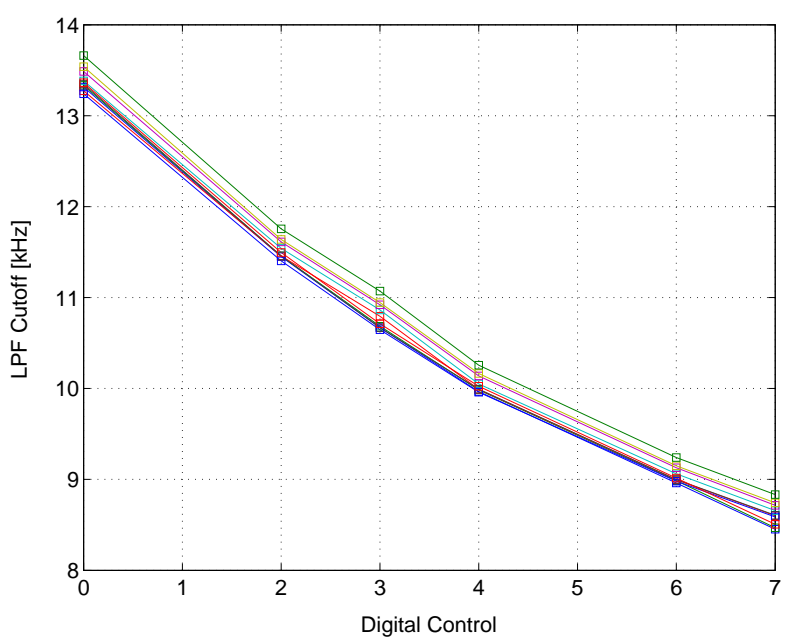

(b)

Fig. 11. Measurements of (a) SPK gains vs. digital control setting, (b) LPF cutoff frequency vs. digital control setting. Curves represent results measured on several dies.

(LFP) and high-frequency spike data, band limited by a programmable-cutoff LPF. Another programmable cutoff filter eliminates the DC component at the input. Amplifier offsets are compensated by means of calibration DACs. The SPK and LFP channels provide variable amplification rates of up to 5000 and 500 , respectively. Input referred noise of $3 \mu \mathrm{V}$ was measured on the SPK channel and $10 \mu \mathrm{V}$ on the LFP channel. The two outputs per each channel are converted into digital signals, and the digital controller produces a serial stream at up to $8 \mathrm{Mbps}$. The controller can also apply a threshold filter to suppress inactive portions of the signal and emit only spike segments, thus potentially reducing the required communication bandwidth. A prototype of the processor has been fabricated on a $0.35 \mu \mathrm{m}$ CMOS process and tested successfully, both electrically and in-vivo. An FPGA board incorporating an embedded CPU core providing for connectivity between the recording processor and a computer host have been developed along with appropriate real-time software.

Thanks to digitizing the recorded signal, separating spikes from LFP and detecting threshold crossings, and thanks to its

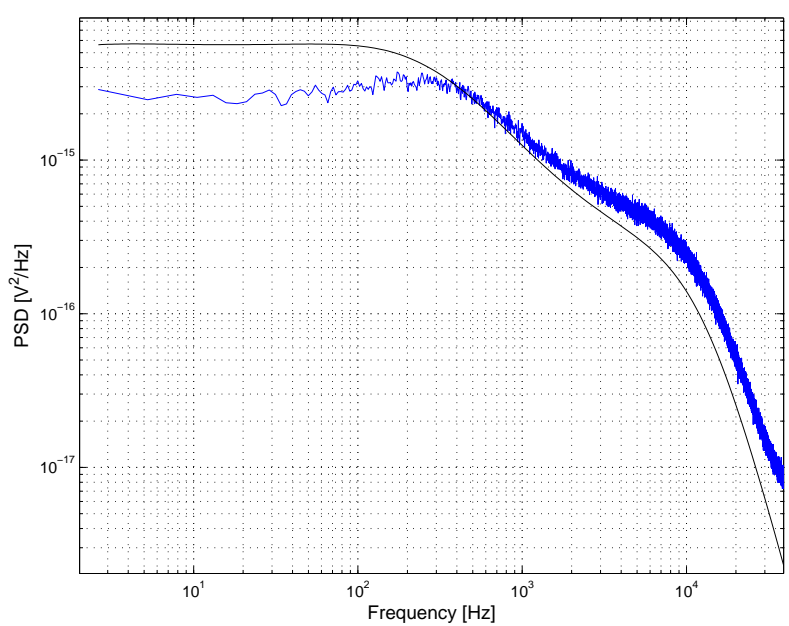

(a)

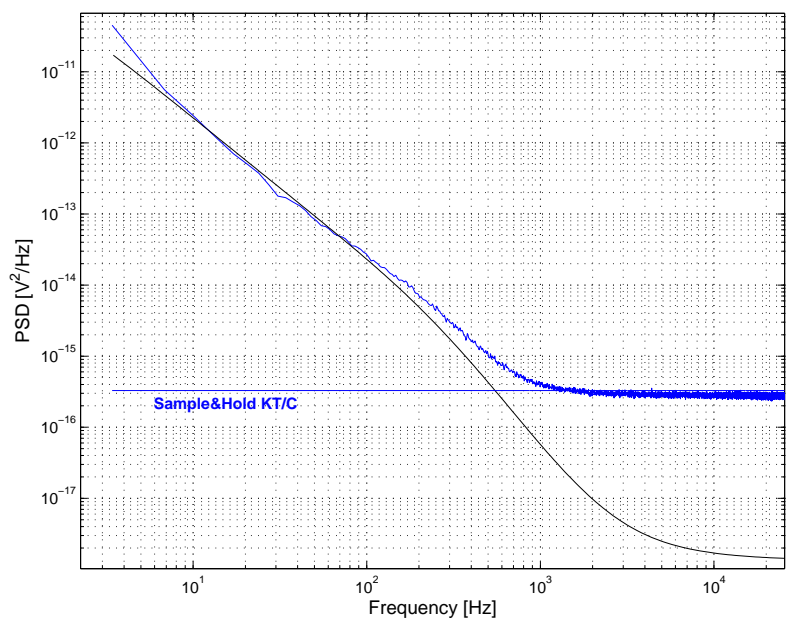

(b)

Fig. 12. Input-referred noise power spectra: (a) SPK. (b) LFP.

programmability, the processor enables digital transmission of only the active spike segments, thus minimizing the required communication bandwidth and allowing for low-power wireless operation.

\section{ACKNOWLEDGEMENT}

This research was funded in part by a grant from the Office of the Chief Scientist, Israel Ministry of Industry and Trade. In-vivo testing has been conducted by D. Anderson, D. Kipke, R. Parikh and K.C. Kong at the University of Michigan.

\section{REFERENCES}

[1] Q. Bai, K. D. Wise, and D. J. Anderson, "A high-yield microassembly structure for three-dimensional microelectrode arrays," IEEE Trans. Biomed Eng., vol. 47, no. 3, pp. 281-289, March 2000.

[2] E. M. Maynard, C. T. Nordhausen, and R. Normann, "The Utah intracortical electrode array: a recording structure for potential brain-computer interfaces," Electroencephalography and clinical Neurophysiology, vol. 102, pp. 228-239, 1997.

[3] А. B. Schwarz, "Cortial neural prosthetics," Annual review of Neuroscience, vol. 27, pp. 487-507, 2004.

[4] M. Nicolelis, "Actions from thoughts," Nature, vol. 409, pp. 403-407, 2001. 


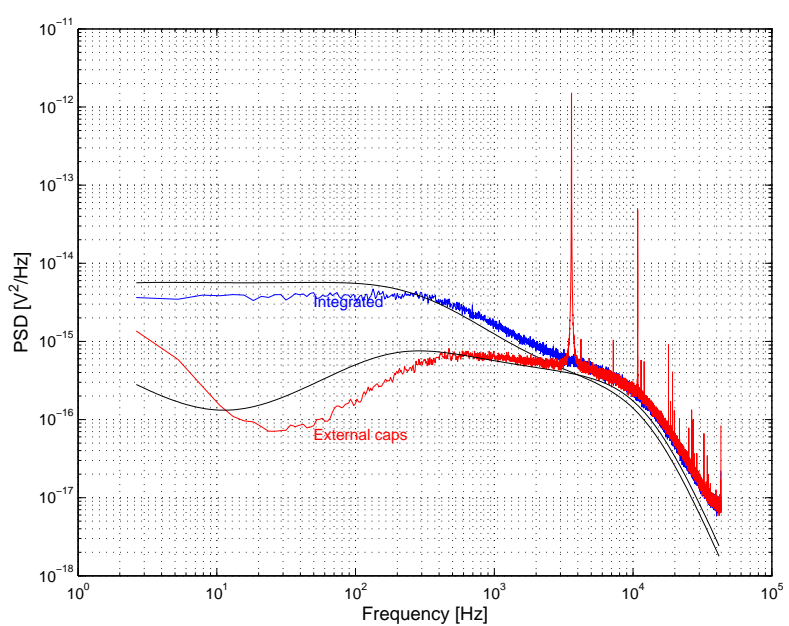

Fig. 13. Input-referred noise power spectra for integrated preamplifier and preamplifier with external elements

[5] M. Black, M. Serruya, E. Bienenstock, Y. Gao, W. Wu, and J. Donoghue, "Connecting brains with machines: the neural control of $2 \mathrm{~d}$ cursor movement," in First International IEEE EMBS Conference on Neural Engineering, 2003.

[6] R. R. Harrison, "A low-power integrated circuit for adaptive detection of action potentials in noisy signals," in Proc. of the 25-th Annual International Conference of the IEEE EMBS, September 2003, pp. 33253328.

[7] K. Oweiss, K. Thomson, and D. Anderson, "A systems approach for real-time data compression in advanced brain-machine interfaces," in Proc. of 2nd Int. IEEE EMBS Conf. Neural Eng., March 2005, pp. 6265.

[8] Y. Perelman and R. Ginosar, "Analog frontend for multichannel neuronal recording system with Spike and LFP separation," Journal of Neuroscience Methods, vol. 153, p. 2126, 2006.

[9] Z. S. Zumsteg, R. E. Ahmed, G. Santhanam, K. V. Shenoy, and T. H. Meng, "Power feasibility of implantable digital spike-sorting circuits for neural prosthetic systems," in Proc. of the 26-th Annual International Conference of the IEEE EMBS, September 2004, pp. 4237-4240.

[10] A. Zviagintsev, Y. Perelman, and R. Ginosar, "Low power architectures for spike sorting," in Proc. of 2nd Int. IEEE EMBS Conf. Neural Eng., March 2005, pp. 162-165.

[11] _ - "Low power spike detection and alignment algorithm," in Proc. of 2nd Int. IEEE EMBS Conf. Neural Eng., March 2005, pp. 317-320.

[12] T. Horiuchi, T. Swindell, D. Sander, and P. Abshire, "A low-power CMOS neural amplifier with amplitude measurements for spike sorting," in ISCAS, 2004.

[13] D. A. Borkholder, "Cell based biosensors using microelectrodes," Ph.D dissertation, Univ. of Stanford, Novermber 1998.

[14] K. Guillory and R. Normann, "A 100-channel system for real time detection and storage of extracellular spike waveforms," Journal of Neuroscience Methods, vol. 91, pp. 21-29, 1999.

[15] I. Obeid, J. C.Morizio, M. A. L. N. K. A. Moxon, and P. D. Wolf, "Two multichannel integrated circuits for neural recording and signal processing," IEEE Trans. on Biomed. Eng., vol. 50, no. 2, pp. 255-258, February 2003.

[16] A. Arieli, A. Sterkin, A. Grinvald, and A. Aertsen, "Dynamics of ongoing activity: Explanation of the large variability in evoked cortical responses," Science, vol. 273, pp. 1868-1871, September 1996.

[17] J. P. Donoghue, J. N. Sanes, N. G. Hatsopoulos, and G. Gaal, "Neural discharge and local field potential oscillations in primate motor cortex during voluntary movements," The Journal of Neurophysiology, vol. 79, no. 1, pp. 159-173, January 1998.

[18] C. Mehring, J. Rickert, E. Vaadia, S. de Oliveira, A. Aertsen, and S. Rotter, "Inference of hand movements from local field potentials in monkey motor cortex," Nature Neuroscience, vol. 6, no. 12, pp. $1253-$ 1254, 2003.

[19] R. R. Harrison and C. Charles, "A low-power low-noise CMOS amplifier for neural recording applications," IEEE Journal of Solid State Circuits, vol. 38, no. 6, pp. 958-965, June 2003.

[20] W. R. Patterson, Y. K. Song, C. W. Bull, I. Ozden, A. P. Deangellis,

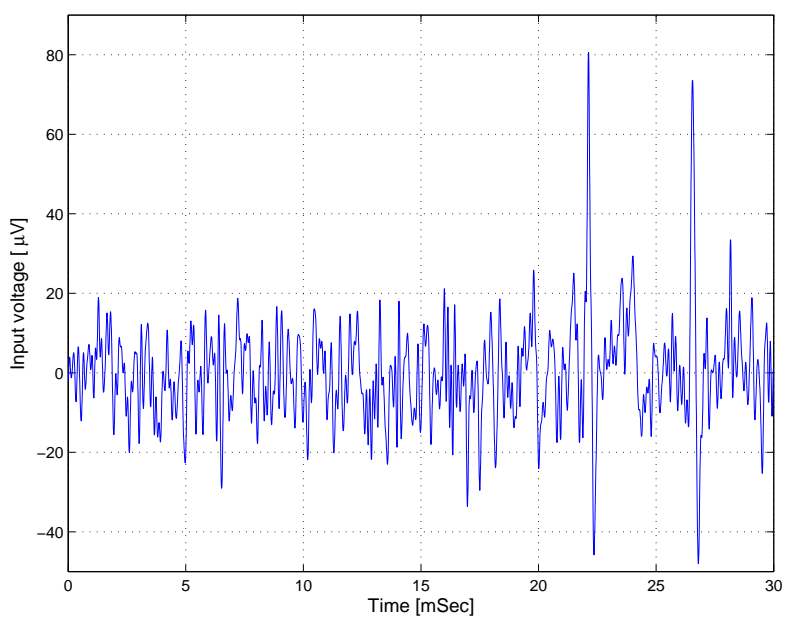

(a)

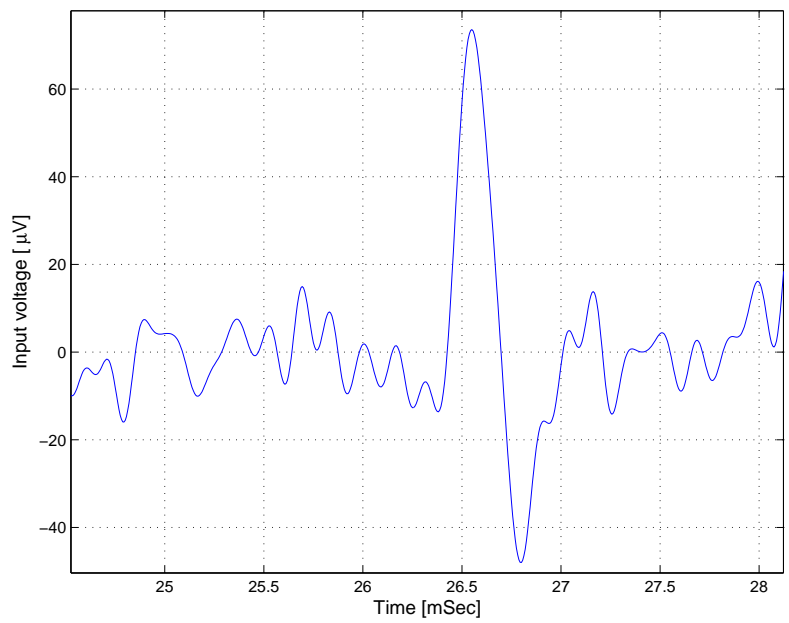

(b)

Fig. 14. (a) Signal recorded from a rat with Michigan probes (b) Closeup.

C. Lay, J. L. McKay, A. V. Nurmikko, J. D. Donoghue, and B. W. Connors, "A microelectrode/microelectronic hybrid device for brain implantable neuroprosthesis applications," IEEE Trans. on Biomed. Eng., vol. 51, no. 10, October 2004.

[21] P. Mohseni and K. Najafi, "A fully integrated neural recording amplifier with DC input stabilization," IEEE Trans. on Biomed. Eng., vol. 51, no. 5, May 2001.

[22] T. Instruments. (2004) TMS320C6000 DSP multichannel buffered serial port (McBSP) reference guide. [Online]. Available: http://ti.com

[23] T. L. Deliyannis, Y. Sun, and J. K. Fidler, Continuous-Time Active Filter Design. CRC Press, 1999.

[24] Y. Perelman and R. Ginosar, "A low power inverted ladder d/a converter," IEEE TCAS II, to be published in, 2006.

[25] J. Morizio, D. Won, I. Obeid, M. Nicolelis, and P. Wolf, "16-channel neural pre-conditioning device," in Proc. of the 1st Int. IEEE EMBS Conf. Neural Eng., March 2003, pp. 104-107.

[26] Q. Bai and K. Wise, "Single-unit neural recording with active microlectrode arrays," IEEE Trans. Biomed. Eng., vol. 48, no. 8, pp. 911-920, August 2001.

[27] R. Olsson, M. Gulari, and K. Wise, "A fully-integrated bandpass amplifier for extracellular neural recording," in Proc. of the 1st Int. IEEE EMBS Conf. Neural Eng., March 2003, pp. 165-168.

[28] D. Chen, J. Harris, and J. Principe, "A bio-amplifier with pulse output," in 26th Ann. Intrn. Conf. IEEE EMBS, Proc., September 2004.

[29] M. Steyaert, W. Sansen, and A. Zhongyuan, "A micropower lownoise monolithic instrumentation amplifier for medical purposes," IEEE Journal of Solid State Circuits, vol. 22, no. 6, pp. 1163-1168, 1987. 